\title{
Effects of a n-3 polyunsaturated fatty acid-enriched diet on embryo production in dairy cows
}

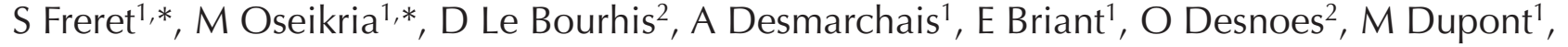 \\ L Le Berre ${ }^{2}$, O Ghazouani ${ }^{1}$, P S Bertevello ${ }^{1}$, A P Teixeira-Gomes ${ }^{3,4}$, V Labas ${ }^{1,3}$, S Uzbekova ${ }^{1}$, \\ P Salvetti ${ }^{2}, \vee$ Maillard ${ }^{1}$ and S Elis ${ }^{1}$ \\ ${ }^{1} P R C$, CNRS, IFCE, INRA, Université de Tours, Nouzilly, France, ${ }^{2}$ Allice, Station de Phénotypage, Nouzilly, France, \\ ${ }^{3}$ PAIB (Pôle d'Analyse et d'Imagerie des Biomolécules), Plate-forme CIRE (Chirurgie et Imagerie pour la Recherche \\ et I'Enseignement), INRA, Université de Tours, CHRU de Tours, Nouzilly, France and ${ }^{4}$ UMR ISP, INRA 1282, \\ Université de Tours, Nouzilly, France
}

Correspondence should be addressed to S Elis; Email: sebastien.elis@inra.fr

*(S Freret and M Oseikria contributed equally to this work)

\begin{abstract}
Beneficial effects of $\mathbf{n}-3$ polyunsaturated fatty acid (PUFA) supplementation on dairy cow reproduction have been previously reported. The objectives of the present study were to assess whether $\mathrm{n}-3$ PUFA supplementation would affect in vitro embryo production (IVP) after ovarian stimulation. Holstein cows received a diet with $\mathbf{1} \%$ dry matter supplementation of either $\mathrm{n}-\mathbf{3}$ PUFA ( $n=18$, microencapsulated fish oil) or a control, n-6 PUFA ( $n=19$, microencapsulated soy oil). Both plasma and follicular fluid FA composition showed integration of total PUFA through the diet. All cows underwent an IVP protocol consisting of ovarian stimulation, ultrasound-guided transvaginal oocyte retrieval (ovum pick-up, OPU, five per cow) followed by in vitro maturation, fertilisation and 7 days of embryo development. A tendency toward an increase in the blastocyst rate (diet effect, $P=0.0865)$ was observed in $n-3$ cows, with $49.6 \pm 5.5 \%$ vs $42.3 \pm 5.5 \%$ in control $n-6$ cows. A significant increase (diet effect, $P=0.0217)$ in the good-quality blastocyst rate (freezable blastocysts) was reported in $n-3$ cows $(42.2 \pm 7.7 \%)$ compared to control $n-6$ cows $(32.7 \pm 7.7 \%)$. A significant difference in lipid composition was shown in the oocytes recovered by OPU from n-3 and n-6 treated cows, by intact single-oocyte MALDI-TOF mass spectrometry. The 42 differentially abundant identified lipids were mainly involved in cell membrane structure. In conclusion, n-3 PUFA supplementation enhanced oocyte quality and modified their lipid composition. Further studies are necessary to investigate the potential link of these lipid modifications with enhanced oocyte quality. Reproduction (2019) 158 71-83
\end{abstract}

\section{Introduction}

Reproductive performance in cows is influenced by nutritional and metabolic status, as previously reviewed (Butler 2000, Roche 2006, Leroy et al. 2008). Appropriate plasma fatty acids (FAs) and polyunsaturated FA (PUFA) composition affects various reproductive processes, including steroid hormones and prostaglandin precursor syntheses via cholesterol and arachidonic acid, respectively (Urlep and Rozman 2013, Tessaro et al. 2015). Previous studies reported beneficial effects of n-3 PUFA supplementation on dairy cow reproduction (reviewed in Moallem 2018). The n-3 PUFA belongs to a family of biologically active essential FA. Given the poor conversion rate between the n-3 PUFA short-chain alpha-linolenic acid (ALA, C18:3) and the long-chain n-3 PUFA (LC n-3 PUFA), eicosapentaenoic acid (EPA, C20:5) and docosahexaenoic acid (DHA, C22:6), these LC n-3 PUFA need to be provided directly through the diet in order to increase their abundance in the plasma (Simopoulos 2002, Plourde and Cunnane 2007, Calder 2012). EPA and DHA are mainly found in fish oil, as well as in microalgae oil (Abughazaleh et al. 2009).

The n-3 PUFA have beneficial effects on cow reproduction, in several ways. Indeed, cows being fed a flaxseed diet had a higher pregnancy rate in relation to a decrease in embryo mortality (Petit \& Twagiramungu 2006), as well as an increase in the size of pre-ovulatory (Ambrose et al. 2006) and small follicles (Zachut et al. 2010). Conception rates also tended to be higher in cows receiving an ALA-enriched diet (Ambrose et al. 2006, Dirandeh et al. 2013) or a LC n-3 PUFA diet (Elis et al. 2016b, Sinedino et al. 2017). One reported mechanism leading to increased conception rates was the uterine effect of n-3 PUFA reducing prostaglandin F2 alpha (PGF2 $\alpha$ ) and, therefore, reducing embryo mortality (Mattos et al. 2004). Nevertheless, other studies suggested a direct beneficial ovarian effect of LC n-3 
PUFA. Indeed, in vitro embryo production, performed after ultrasound-guided transvaginal oocyte retrieval (ovum pick-up) without ovarian stimulation treatment, was enhanced after n-3 PUFA supplementation compared to the control, consisting of a saturated FA supplementation (Moallem et al. 2013). Moreover, in vitro studies also reported that in vitro maturation (IVM) medium supplemented with either ALA (Marei et al. 2009) or low-dose DHA (Oseikria et al. 2016) led to increased embryo developmental rates. Since previous studies also reported that n-6 PUFA could lead to beneficial effects on reproduction (reviewed in Leroy et al. 2014), it is therefore important to compare n-3 PUFA supplementation to a $\mathrm{n}-6$ control, in order to prove a specific n-3 PUFA effect. Indeed, when comparing n-3 PUFA to a saturated control, any beneficial effect could be due to the difference between saturated and polyunsaturated FA, which is not specific to n-3 PUFA.

The present study relies on the hypothesis that $n-3$ PUFA supplementation of the diet of donor cows could improve embryo production both in terms of number and quality of embryos after ovarian stimulation. The objectives of the present study in dairy cows were therefore (1) to assess whether dietary supplementation with LC n-3 PUFA (in comparison with a n-6 PUFA control diet) would affect in vitro embryo production, performed after ovarian stimulation by gonadotrophins (FSH treatment), thus mimicking usual in vitro or in vivo embryo production conditions; (2) to assess several LC n-3 PUFA supplementation durations in order to determine whether a short duration could be long enough to observe beneficial effects of the diet on embryo production and (3) to analyse oocyte lipid composition after LC n-3 PUFA diet supplementation.

\section{Materials and methods \\ Experimental design}

All experimental protocols were conducted in accordance with the European Directive 2010/63/EU on the protection of animals used for scientific purposes and approved by the French Ministry of National Education, Higher Education, Research and Innovation after ethical assessment by the local ethics committee 'Comité d'Ethique en Expérimentation Animale Val de Loire (CEEA VdL)' (protocol registered under ref. APAFIS\#2098-2015100115278976_v5).

Holstein primiparous cows $(n=37)$ were managed in loose housing during lactation. In order to conclude that the observed effects were specifically due to n-3 PUFA, cows supplemented with n-6 PUFA were used as a control group. Each cow received either n-3 $(n=18)$ or n-6 $(n=19)$ supplementation for 9 weeks, starting at $\approx 11$ weeks postpartum. All cows underwent five sessions of OPU. Three of these OPU sessions, occurring after ovarian stimulation (FSH treatment), led to in vitro embryo production, after 2, 5 and 7 weeks of supplementation. The two other OPU sessions led to oocyte collection and lipidomic analyses, after 3 weeks (without ovarian stimulation) and 9 weeks (with ovarian stimulation) of supplementation (Fig. 1).

\section{Experimental diets and dry matter intake}

For 9 weeks, dairy cows received a diet consisting of (\% of total diet dry matter, DM): $59.4 \%$ corn silage, $14.5 \%$ Sandilait $\mathbb{R}$ (soybean and rapeseed meal; Agralys, Blois, France), 9.5\% Sandifort ${ }^{\circledR}$ (wheat; Agralys, Blois, France), $8.5 \%$ alfalfa hay, $7.5 \%$ dehydrated alfalfa, $0.2 \%$ vitamins and minerals $5 / 23$ and $0.4 \%$ calcium bicarbonate. The n-3 PUFA supplement was OMG750®, a microencapsulated fish oil (Kemin, Nantes, France) and the n-6 PUFA supplement was OMGSOY ${ }^{\circledR}$, a microencapsulated soy oil (Kemin, Nantes, France), with both supplements being distributed at 1\% DM. The two diets were total mixed rations (TMR), with feed values calculated using the INRA French feeding system (INRA 2018). The supplements were manually mixed into the diet and the TMR were distributed twice daily in individual weighing troughs (Insentec B.V., Marknesse, The Netherlands), as described by Elis et al. (2016b). Cows were fed ad libitum and DM intake (DMI) was determined from the feed intake, the composition of the diets and the dry matter content of each ingredient included in the diets. The nutritional values of the different ingredient compositions in the diets were calculated from a chemical analysis. DMI was analysed daily during the distribution of the $n-3$ or $n-6$ diets, throughout the supplementation period.

\section{Body weight, milk yield and energy balance}

Body weight (BW) and milk yield (MY) were monitored daily during the supplementation period. All cows were milked twice daily. In the milking parlour, the MY $(\mathrm{kg} / \mathrm{d})$ of each cow was automatically recorded (software Manufeed 500 pro, vc5 version 2.011.14). After each milking period, cows were automatically weighed (software RIC version RW1.7). Only the BW recorded in the morning was used for statistical analysis.

Energy balance (EB, expressed in Mcal/day) was calculated during the supplementation period and corresponds to the difference between net energy intake and net energy needs for body maintenance and lactation, according to the INRA method (INRA 2018).

\section{AMH and metabolic assays, fatty acid composition of plasma and follicular fluid}

Plasma anti-Müllerian hormone $(\mathrm{AMH})$ was determined using the AMH Gen II ELISA assay (Beckman Coulter) using $50 \mu \mathrm{L}$ of undiluted plasma, as previously described (Rico et al. 2009), from samples collected the first day of the diet supplementation and stored at $-20^{\circ} \mathrm{C}$. $\mathrm{AMH}$, an indicator of the ovarian response after hormonal stimulation, was assessed for any differences in plasma AMH between the groups.

For metabolic assays, plasma samples were collected once per week from caudal venipuncture before feeding, during the supplementation period. Plasma samples were stored at $-20^{\circ} \mathrm{C}$ until the assays were conducted. Plasma NEFA, urea and glucose were determined using enzymatic colorimetry with a multi-parameter analyser (KONE Instruments Corporation, Espoo, Finland). 

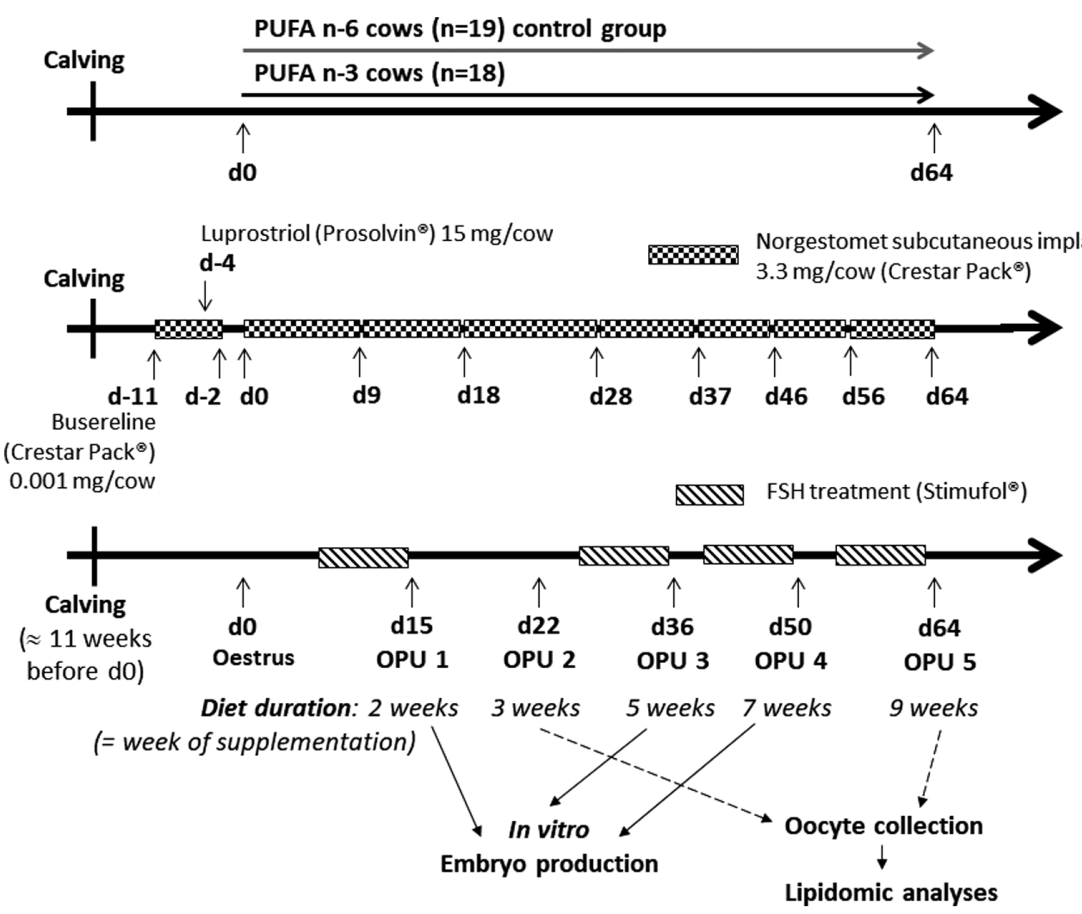

Figure 1 Experimental design. Thirty-seven cows underwent five ovum pick-up (OPU) sessions to either collect oocyte-cumulus complexes and perform in vitro embryo production (OPU sessions 1, 3 and 4) or to collect oocytes and perform lipid composition mass spectrometry analysis (OPU sessions 2 and 5). Cows received a diet supplemented with either PUFA n-3 or PUFA n-6 supplementation during 9 weeks, beginning ( $=\mathrm{d} 0, \mathrm{~d}$ stands for day) on average 11 weeks postpartum. Each cow received a synchronisation treatment, eleven days prior to $\mathrm{d} 0$, consisting of a $3.3 \mathrm{mg}$ norgestomet subcutaneous implant and an intra muscular (IM) injection of $0.001 \mathrm{mg}$ buserelin (Crestar Pack ${ }^{\circledR}$, MSD Santé Animale, Beaucouzé, France). Seven days later (d-4), each cow received an IM injection of $15 \mathrm{mg}$ luprostriol (Prosolvin $®$, Virbac, Carros, France) and 2 days later ( $\mathrm{d}-2)$, the subcutaneous implant was removed. Oestrus was observed $\approx 48 \mathrm{~h}$ following implant removal $(\mathrm{d}=0)$, and a new subcutaneous implant of $3.3 \mathrm{mg}$ norgestomet was inserted on the same day (Crestar ${ }^{\circledR} S \mathrm{SO}$, MSD Santé Animale, Beaucouzé, France). The norgestomet implant was then replaced every 9 or 10 days until the end of the experiment (d64, last day of supplementation), to prevent the occurrence of oestrus between OPU sessions. A FSH treatment (ovarian stimulation with Stimufol $\mathbb{\circledR}$ (Reprobiol, Ouffet, Belgium) was performed before OPU 1, OPU 3, OPU 4 and OPU 5.
Plasma and follicular fluid fatty acid compositions were determined using gas chromatography, as previously described (Lefils et al. 2010). Plasma fatty acid composition was determined at the beginning of the supplementation period and in samples corresponding to OPU leading to in vitro embryo production (at 2, 5 and 7 weeks postpartum, Fig. 1). Follicular fluid fatty acid composition was determined at 2, 5 and 7 weeks postpartum.

\section{Embryo production}

\section{Oestrus synchronisation and ovarian stimulation}

Eleven days prior to supplementation (=d0, Fig. 1), each cow received a synchronisation treatment consisting of a $3.3 \mathrm{mg}$ norgestomet subcutaneous implant and an intra muscular (IM) injection of $0.001 \mathrm{mg}$ buserelin (Crestar Pack®, MSD Santé Animale, Beaucouzé, France). Seven days later, each cow received an IM injection of $15 \mathrm{mg}$ luprostriol (Prosolvin $\AA$, Virbac, Carros, France) and 2 days later, the subcutaneous implant was removed. Oestrus was observed $\approx 48 \mathrm{~h}$ following implant removal, which corresponded to the first day of supplementation, and a new subcutaneous implant of $3.3 \mathrm{mg}$ norgestomet was inserted this same day (Crestar $\AA$ SO). The norgestomet implant was then replaced every 10 days during the 9 weeks of the experiment, until the end of the protocol, to prevent the occurrence of oestrus between OPU sessions.

Ovarian stimulation was performed using $400 \mu \mathrm{g}$ of $\mathrm{pFSH}$ per cow (Stimufol $®$, Reprobiol, Ouffet, Belgium) divided into five IM injections of decreasing FSH doses $(112,100,75,63$ and $50 \mu \mathrm{g})$ every $12 \mathrm{~h}$, beginning $60 \mathrm{~h}$ prior to the follicular punctures.

\section{Ovum pick-up}

The ultrasound-guided transvaginal oocyte retrieval (ovum pick-up) was performed under locoregional anaesthesia (epidural block) with $86.5-173 \mathrm{mg}$ of procaine per cow (Procamidor $\AA$, Richter Pharma, Wels, Austria), after sedation with $10 \mathrm{mg} / 100 \mathrm{~kg}$ xylazine (Rompun $®$, Bayer Animal Health, Saint-Georges-de-Reneins, France). The anogenital area was then washed and disinfected (Vétédine savon ${ }^{\circledR}$, Vétoquinol, Lure, France). A guide containing an ultrasonographic probe was inserted via the vaginal route and the ovary was positioned by the technician through the transrectal route against the probe (probe EC123, 7.5 MHz, echograph MyLab30, ESAOTE Pie Medical, Saint-Germain-en-Laye, France). A needle holder, including a needle (18G) and linked to a suction system, was introduced via the transvaginal route. The 37 cows were divided into five groups of 6-9 cows. For each group, one OPU session occurred on the same day. The complete experimental design (groups 1-5) was performed over a 14-month period. 


\section{In vitro embryo production}

Complexes oocyte-cumulus (COC) recovered from OPU sessions, performed after 2, 5 and 7 weeks of supplementation, were selected and underwent in vitro maturation (IVM) followed by fertilisation (IVF). All straws of semen used to perform the IVF were produced from a single ejaculate of a single Holstein bull. The produced zygotes then underwent in vitro development (IVD), according to a previously described protocol (Oseikria et al. 2016, Elis et al. 2017). Quality of the produced embryos was assessed through the cleavage rate at $48 \mathrm{~h}$ (reported to COC in IVM) and the blastocyst rate (reported to cleaved embryos) at 7 days following IVF. Blastocyst quality was also graded, with grades ranging from quality 1 (Q1) to quality 4 (Q4) blastocysts, according to the IETS (International Embryo Technology Society) morphological criteria (International Embryo Transfer Society 2013). Q1 and Q2 blastocysts were considered as good-quality and freezable blastocysts. All embryos have been graded by the same lab technician.

\section{Oocyte lipidomic analyses}

Immature oocytes recovered from OPU sessions performed after 3 and 9 weeks of supplementation, in 9 (from the 18) n-3 cows and 9 (from the 19) $n-6$ cows, were used for lipidomic analyses: a total of 60 n-3 oocytes and 61 n- 6 oocytes were compared.

Immature oocytes were completely mechanically denuded from Cumulus cells (CC) by repeated pipetting and individually snap-frozen in drops of $20 \mathrm{mM}$ Tris- $\mathrm{HCl} \mathrm{pH} 6.8 / 260 \mathrm{mM}$ sucrose buffer. Lipid profiles of individual oocytes were obtained using an UltrafleXtreme MALDI-mass spectrometer in positive reflector mode using 2,5-dihydroxyacetophenone matrix, as described (Elis et al. 2017). M/z peaks were detected in the range of $400-1000 \mathrm{~m} / \mathrm{z}$ and values of the normalised peak heights (NPH) were quantified using Progenesis MALDI $^{\text {TM }}$ (Nonlinear Dynamics). Lipids were identified by high-resolution mass spectrometry by LC-MS or by direct infusion combined with top-down MS/MS analyses (Bertevello et al. 2018).

\section{Statistical analyses}

Statistical analyses were performed using $S A S \circledR$ software (SAS Institute Inc. 2013), unless otherwise indicated. The stages (days in milk) and plasma AMH levels of n-3 and n-6 cows at the beginning of supplementation were compared using a Wilcoxon test (NPAR1WAY procedure). Linear mixed models (MIXED procedure) including the effects of diet, week of supplementation (= rank of OPU session), diet $\times$ week of supplementation interaction, with week of supplementation as a repeated effect within cow (repeated statement of the MIXED procedure, an AR(1) covariance structure was used for all parameters except for plasma and follicular fluid FA composition for which a CS covariance structure was used) and a random group effect were used for the following parameters: BW (with BW at 1 week of supplementation as a covariate), MY (with the day in milk corresponding to the first day of supplementation as a covariate), DMI (with BW as a covariate), EB, plasma NEFA, glucose, urea (with, for each of these three parameters, the plasma level measured on the first day of supplementation as a covariate), plasma and follicular fluid FA composition, number of COC recovered and that underwent IVM, number of embryos produced per OPU session. Logistic regression mixed models (GLIMMIX procedure) including the effects of diet, week of supplementation (=rank of OPU session), diet $\times$ week of supplementation interaction and a random group effect were used for the following parameters: cleavage rates and embryo development rates. Results are presented as least squares means (Ismeans) \pm S.E.M., unless otherwise indicated. Multiple comparisons of Ismeans estimated by the models were performed with a $T$-test, and a Bonferroni adjustement was used for all parameters except COC and embryo production parameters, plasma and follicular fluid FA composition parameters.

Relative lipid abundance in individual oocytes were compared using a $T$-test with a Benjamini-Hochberg correction applied to the NPH values of each lipid species; multivariate principal component analysis (PCA) was performed using differential NPH values (XLSTAT, Addinsoft, Paris, France).

Effects with $P \leq 0.05$ were considered significant, and effects with $0.05<P \leq 0.1$ were considered tendencies.

\section{Results}

\section{Physiological measurements}

Supplementation began at $11.2 \pm 0.3$ weeks after calving, with no difference between the control n-6 and $n-3$ groups $(P=0.9273)$. No difference was reported between $n-3$ and $n-6$ groups for BW, MY, DMI or EB (Fig. 2). During the 9-week supplementation period and the previous 8 weeks, BW averaged $572.3 \pm 3.4 \mathrm{~kg}$ in $\mathrm{n}-3$ cows vs $573.5 \pm 3.3 \mathrm{~kg}$ in $\mathrm{n}-6$ cows (diet effect, $P=0.7421)$. MY averaged $28.5 \pm 1.5 \mathrm{~kg} /$ day in $\mathrm{n}-3$ cows vs $28.8 \pm 1.5 \mathrm{~kg} /$ day in $\mathrm{n}-6$ cows (diet effect, $P=0.8069$ ). During the 9-week supplementation period, DMI averaged $19.2 \pm 1.0 \mathrm{~kg} /$ day in $\mathrm{n}-3$ cows compared to $19.4 \pm 1.0 \mathrm{~kg} /$ day in $\mathrm{n}-6$ cows (diet effect, $P=0.8912$ ). EB was, on average, $0.6 \pm 1.1 \mathrm{Mcal} /$ day in $n-3$ cows vs $1.2 \pm 1.1 \mathrm{Mcal} / \mathrm{d}$ in $\mathrm{n}-6$ cows (diet effect, $P=0.6192$ ). For these four measurements, the effect of week of supplementation was significant $(P \leq 0.05)$, without an interaction between diet and week of supplementation.

\section{Metabolic measurements}

Metabolic measurements, measured in plasma (NEFA, glucose, urea), did not differ between n-3 cows and control n-6 cows during the 9-week supplementation period (Fig. 3). Indeed, n-3 cows exhibited a plasma NEFA level of $0.30 \pm 0.02 \mathrm{mM}$ compared to $0.32 \pm 0.02 \mathrm{mM}$ in the control $n-6$ cows (diet effect, $P=0.2911$ ). The plasma glucose levels were, on average, $4.18 \pm 0.05 \mathrm{mM}$ in $\mathrm{n}-3$ cows vs $4.13 \pm 0.05 \mathrm{mM}$ in control $\mathrm{n}-6$ cows (diet effect, $P=0.4925$ ). The plasma urea levels were 

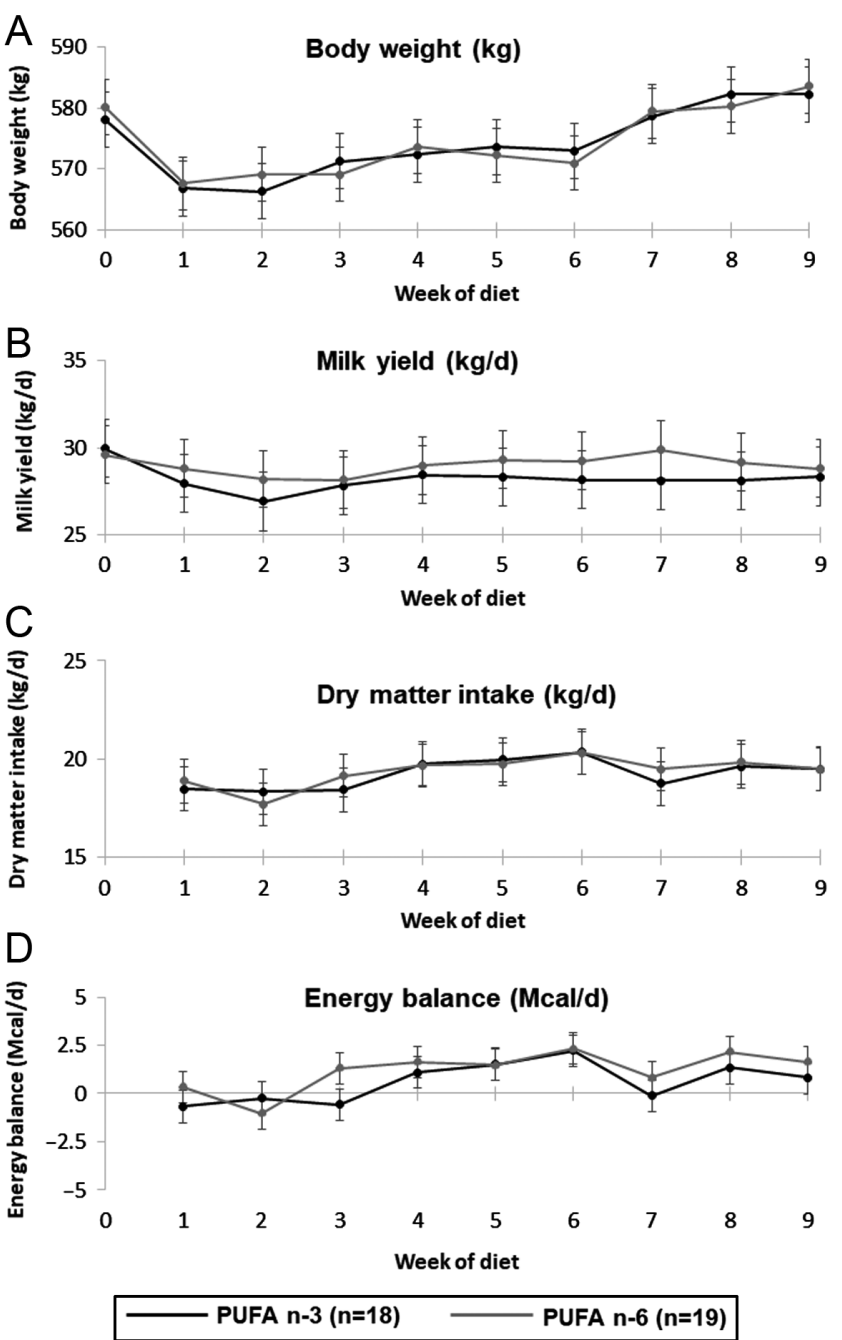

Figure 2 Physiological measurements. Body weight (A), milk yield $(B)$, dry matter intake $(C)$ and energy balance $(D)$ were monitored in 37 primiparous Holstein cows ( $\mathrm{n}-3, n=18 ; \mathrm{n}-6, n=19)$. Cows received either a PUFA n-3 diet (black line) or a PUFA n-6 diet (grey line) for 9 weeks. Results are presented as Ismeans \pm S.E.M. $*$ indicates a difference $(P<0.05) ;{ }^{*}$ indicates a tendency $(P<0.10)$.

not different (diet effect, $P=0.1834$ ) between $\mathrm{n}-3$ cows $(4.23 \pm 0.17 \mathrm{mM})$ and control $\mathrm{n}-6$ cows $(4.40 \pm 0.17 \mathrm{mM})$. For these three measurements, the effect of the week of supplementation was significant $(P \leq 0.05)$, without an interaction between diet and week of supplementation, except for urea level, which had a significant effect of the interaction between diet and week of supplementation $(P=0.025)$.

\section{Plasma and follicular fluid fatty acid composition}

The fatty acid composition, expressed in \% of total fatty acids, was measured in plasma, to assess the efficiency of the PUFA supplementation (Table 1), and in follicular fluid, to study how PUFA nutritional changes affect the fatty acid composition of this ovarian
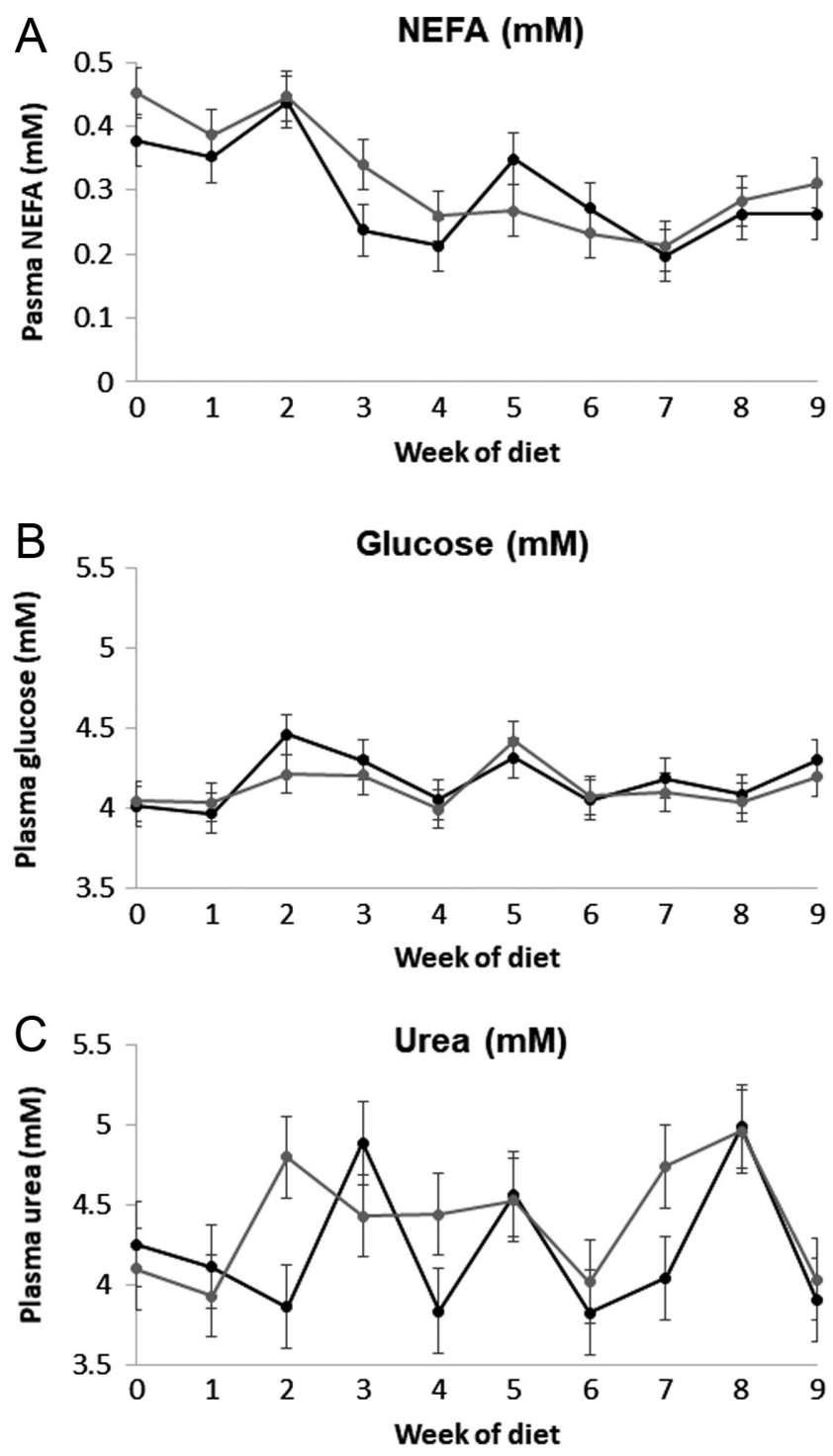

PUFA n-3 (n=18)

PUFA n-6 (n=19)

Figure 3 Metabolic measurements. Plasma NEFA (A), glucose (B) and urea $(C)$ levels were monitored once per week in 37 primiparous Holstein cows ( $n-3, n=18 ; n-6, n=19)$. Cows received either a PUFA n-3 diet (black line) or a PUFA n-6 diet (grey line) for 9 weeks. Results are presented as Ismeans \pm S.E.M. ${ }^{*}$ indicates a difference $(P<0.05) ;{ }^{*}$ indicates a tendency $(P<0.10)$.

compartment (Table 2). Half of the measurements in plasma (15/27) and follicular fluid (10/27) varied throughout the period of supplementation (week of supplementation effect, $P \leq 0.05)$. In n-3 cows, after only 2 weeks of supplementation, a significant two-fold increase was observed in plasma EPA, compared to the period before supplementation $(P<0.0001)$. Similarly, a significant 2.1 -fold increase $(P<0.0001)$ and 1.2 -fold increase $(P<0.0001)$ were also observed for DHA and total n-3 PUFA, respectively, compared to the period before supplementation. The week of supplementation 


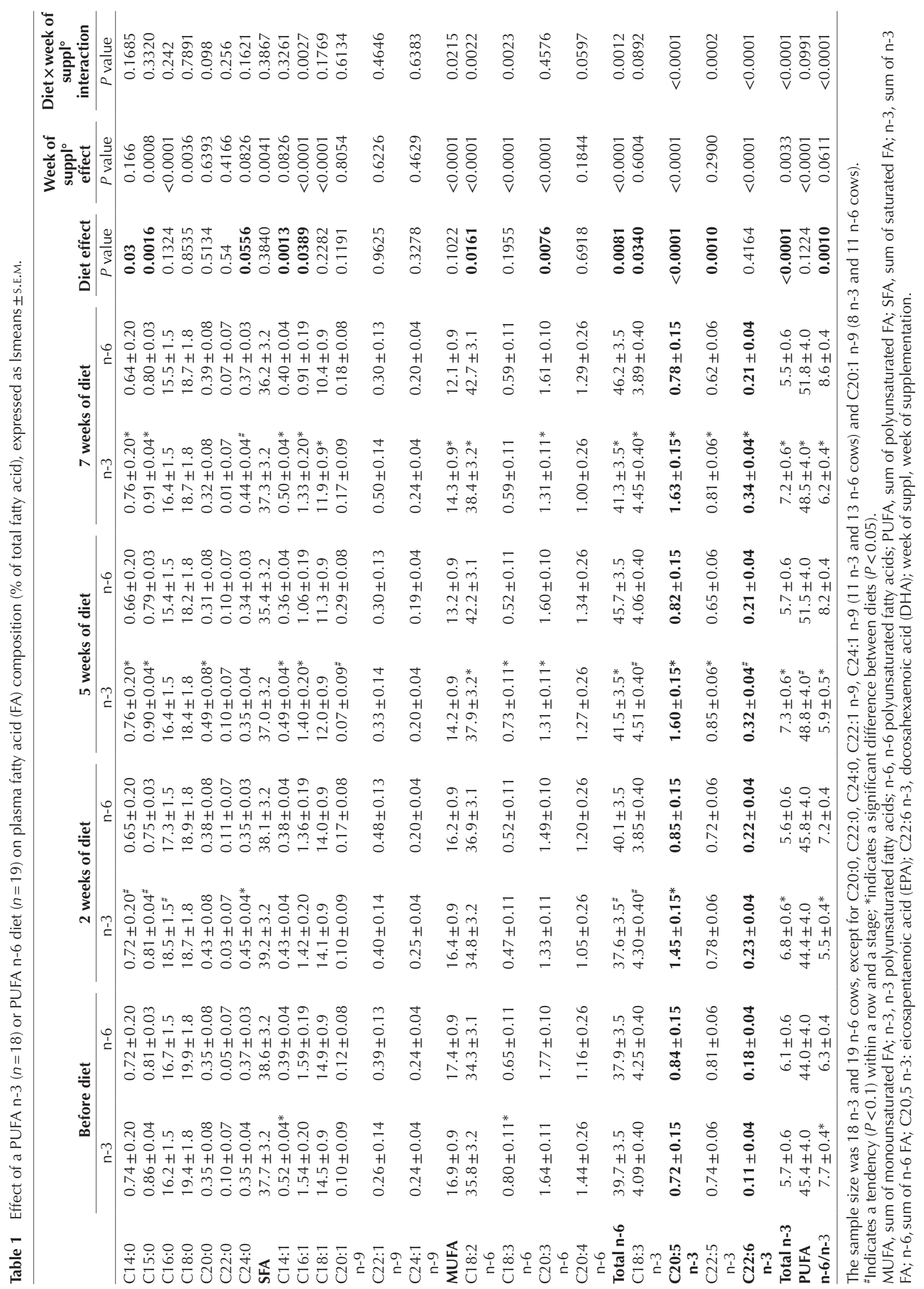




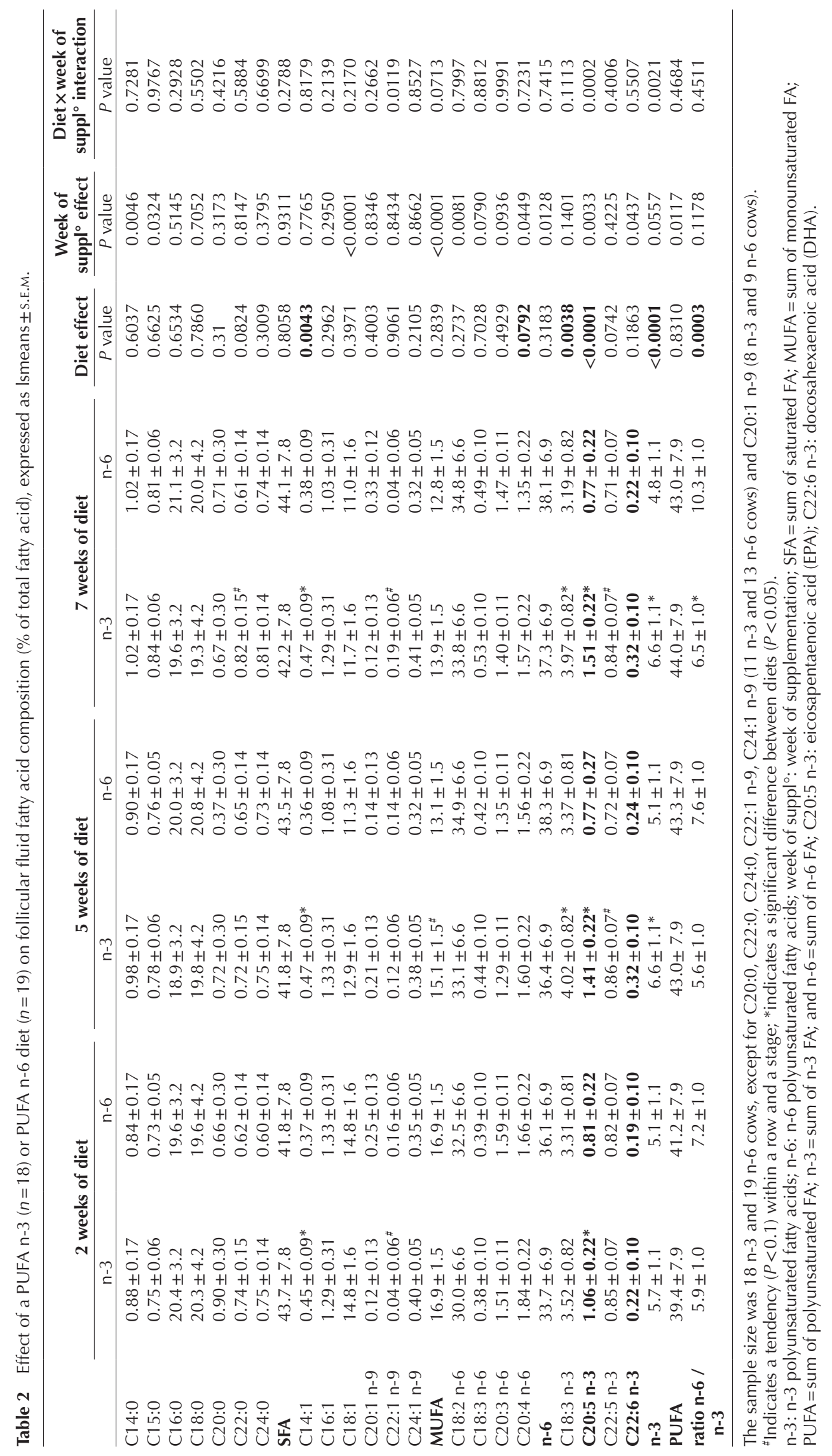


effect was $P<0.0001$ for EPA, $P<0.0001$ for DHA and $P=0.0033$ for total n-3 PUFA; these increases validated the efficiency of the $n-3$ diet. Concerning EPA and total n-3 PUFA, an effect of the diet was reported $(P<0.0001)$ : plasma level at 2 weeks of supplementation was significantly increased for EPA $(1.7$-fold, $P<0.0001)$ and total n-3 PUFA (1.2-fold, $P=0.0002)$ in $n-3$ cows compared to n-6 cows; plasma level at 7 weeks of supplementation was significantly increased for DHA (1.6-fold, $P=0.0298$ ), this increase being a tendency at 5 weeks of supplementation ( 1.5 -fold, $P=0.0534)$, in $n-3$ cows. Moreover, the follicular fluid level also increased by 1.3 -fold for EPA after 2 weeks of supplementation $(P=0.0344)$ and for total n-3 PUFA after 5 weeks of supplementation $(P<0.0001)$ in $\mathrm{n}-3$ cows compared to control n-6 cows. Concerning total n-6 PUFA (diet effect, $P=0.0081$ ), a tendency toward a 1.1 -fold increase was reported in control n- 6 cow plasma at 2 weeks of supplementation $(P=0.0833)$, with this 1.1 -fold increase becoming significant at 5 weeks of supplementation $(P=0.0039)$. In $\mathrm{n}-3$ cows, the $\mathrm{n}-6$ PUFA/n-3 PUFA ratio (diet effect, $P=0.0010$ ) was 1.3 -fold lower in $n-3$ cow plasma at 2 weeks of supplementation $(P=0.0075)$ and 1.6-fold lower in $n-3$ cow follicular fluid at 7 weeks of supplementation $(P=0.0052)$, compared to control $\mathrm{n}-6$ cows. For these five measurements (EPA, DHA, total n-3 PUFA, total n-6 PUFA and n-6/n-3 ratio), the diet $\times$ week of supplementation interaction was significant $(0.0001<P \leq 0.0012)$.

\section{Embryo production}

Three embryo production sessions by OPU-IVF were performed for $18 \mathrm{n}-3$ cows and 19 control n- 6 cows, generating 54 and 57 OPU-IVF sessions and a total of 1462 and 1538 punctured follicles, respectively (Table 3). Plasma AMH level was not different between the groups $(p=0.4941)$, suggesting an absence of a difference in the ovarian response to hormonal stimulation between the groups; it was, on average, $299.0 \pm 73.4 \mathrm{pg} / \mathrm{mL}$ in $\mathrm{n}-3$ cows compared to $242.3 \pm 55.6 \mathrm{pg} / \mathrm{mL}$ in control n- 6 cows. The average number of punctured follicles per OPU session did not differ (diet effect, $P=0.8196$ ) between $n-3$ cows $(26.7 \pm 4.3)$ and control $n-6$ cows (26.1 \pm 4.3$)$.

A significantly higher recovered COC rate (diet effect, $P=0.0035)$ was observed in $\mathrm{n}-3$ cows $(38.0 \pm 1.6 \%)$ compared to control n- 6 cows $(32.8 \pm 1.6 \%)$. This rate corresponded to $10.2 \pm 1.3$ oocytes recovered per OPU session in $\mathrm{n}-3$ cows compared to $8.5 \pm 1.3$ from control n-6 cows (diet effect, $P=0.2297$ ). The cleavage rate tended to be lower $(P=0.1033)$ in $\mathrm{n}-3$ cows $(77.3 \pm 3.8 \%)$ compared to control $n-6$ cows $(82.3 \pm 3.3 \%)$, which corresponded to $5.8 \pm 1.0$ cleaved embryos in $n-3$ cows vs $5.0 \pm 1.0$ in control $n-6$ cows (diet effect, $P=0.3597$ ). The blastocyst rate tended to be higher (diet effect, $P=0.0865)$ in $n-3$ cows $(49.6 \pm 5.5 \%$ ) vs control n-6 cows $(42.3 \pm 5.5 \%)$, corresponding to $2.8 \pm 0.6$ blastocysts per OPU session in $n-3$ cows vs $2.2 \pm 0.6$ blastocysts in control $n-6$ cows (diet effect, $P=0.2136)$. This tendency was observed only during the first OPU session $(P=0.1024)$, therefore, only after 2 weeks of supplementation. The quality of blastocysts was also morphologically graded, into good-quality embryos $(\mathrm{Q} 1$ and Q2 according to the IETS classification, considered as freezable embryos) and lower quality embryos (Q3 and Q4). A significant increase (diet effect, $P=0.0217$ ) in the Q1-Q2 blastocyst rate was noted in $\mathrm{n}-3$ cows $(42.2 \pm 7.7 \%)$ compared to control n-6 cows $(32.7 \pm 7.7 \%)$, which corresponded to $2.5 \pm 0.5$ Q1-Q2 blastocysts in $\mathrm{n}-3$ cows vs $1.8 \pm 0.5$ Q1-Q2 blastocysts in control n-6 cows (diet effect, $P=0.1527)$. This difference was significant after 5 weeks of supplementation $(P=0.0447)$ and a trend after 2 weeks of supplementation $(P=0.0773)$. No difference was observed after 7 weeks of supplementation between the diet groups, for any parameter. Indeed, in n-3 cows, embryo development rates were maintained at the same levels as for the previous OPU-IVF session (after 2 and 5 weeks of dietary supplementation), but were no longer different from the developmental rates of control n-6 cows. Concerning embryo development rates, no parameter showed neither a significant week of supplementation effect, except for the recovered COC rate $(P=0.0054)$, nor a significant diet $\times$ week of supplementation interaction. Concerning the number of embryos produced per OPU session, no parameter showed a significant week of supplementation effect or a significant diet $\times$ week of supplementation interaction.

\section{Oocyte lipid composition}

A total of 60 single oocytes from 9 n-3 cows (n-3 oocytes) and 61 oocytes from 9 n- 6 cows (n-6 oocytes) were analysed by intact cell MALDI-TOF mass spectrometry, to compare their lipid composition. Physiological and metabolic measurements for these two subgroups (9 n-3 and 9 n- 6 cows) were representative of the whole groups (respectively $18 \mathrm{n}-3$ and $19 \mathrm{n}-6$ cows) (Supplementary Fig. 1 and Supplementary Tables 1, 2, see section on supplementary data given at the end of this article). Hierarchical clustering was performed, based on $110 \mathrm{~m} / \mathrm{z}$ that were differentially abundant between $\mathrm{n}-3$ and $\mathrm{n}-6$ oocytes $(P<0.05,>2$-fold change, Fig. 4A). A heatmap represents the relative abundance of 55 upregulated and 55 downregulated $\mathrm{m} / \mathrm{z}$ in the $\mathrm{n}-3$ group as compared to the $\mathrm{n}-6$ group (Fig. $4 \mathrm{~A}$ and Supplementary Table 3); each line corresponds to one oocyte and each column corresponds to one differential molecular species. Two clear clusters were distinguished, corresponding to overabundant lipids in either $n-3$ or $n-6$ oocytes. A principle component analysis performed on differential $\mathrm{m} / \mathrm{z}$ clearly discriminated $\mathrm{n}-3$ oocytes and control n-6 oocytes, based on their lipid 


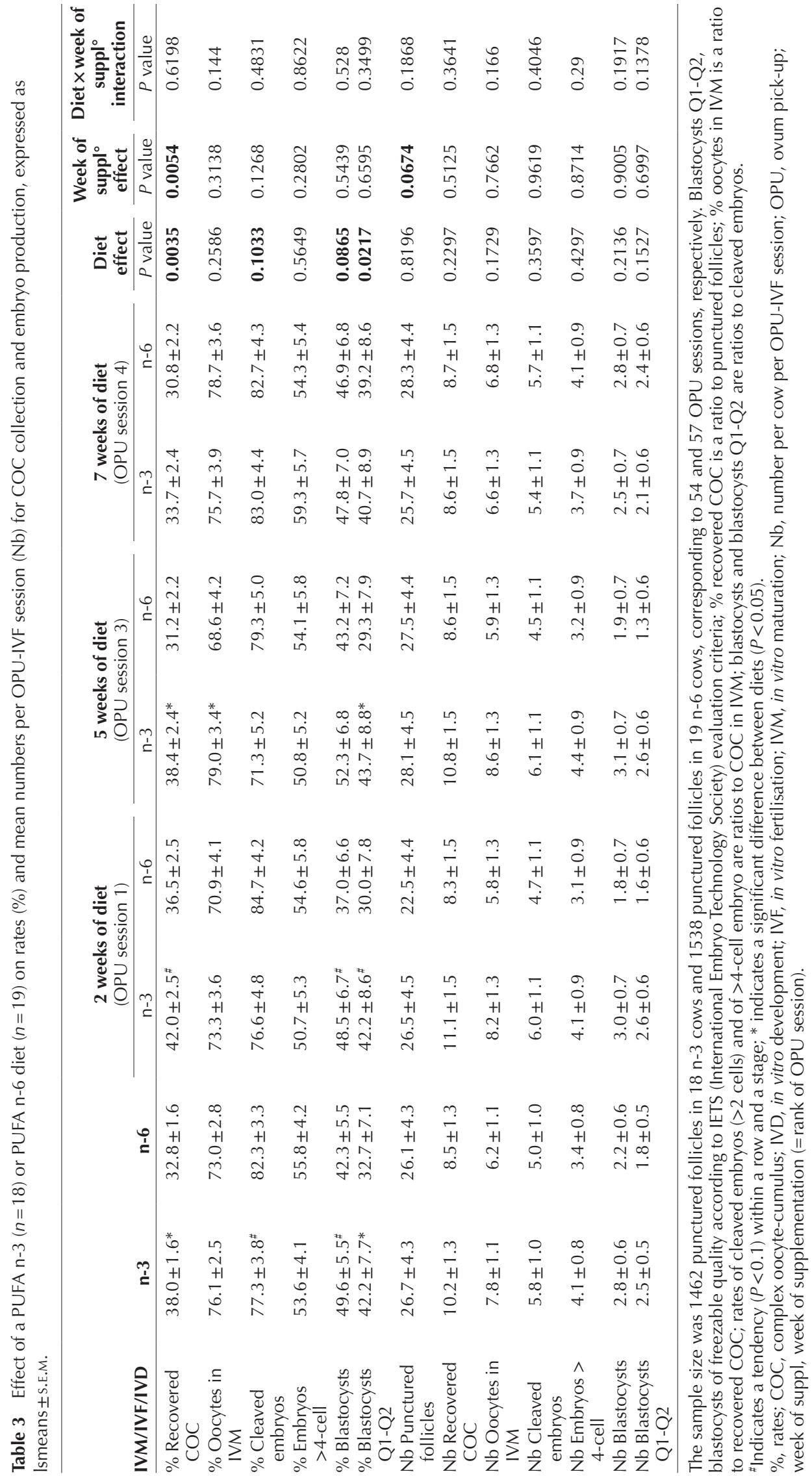



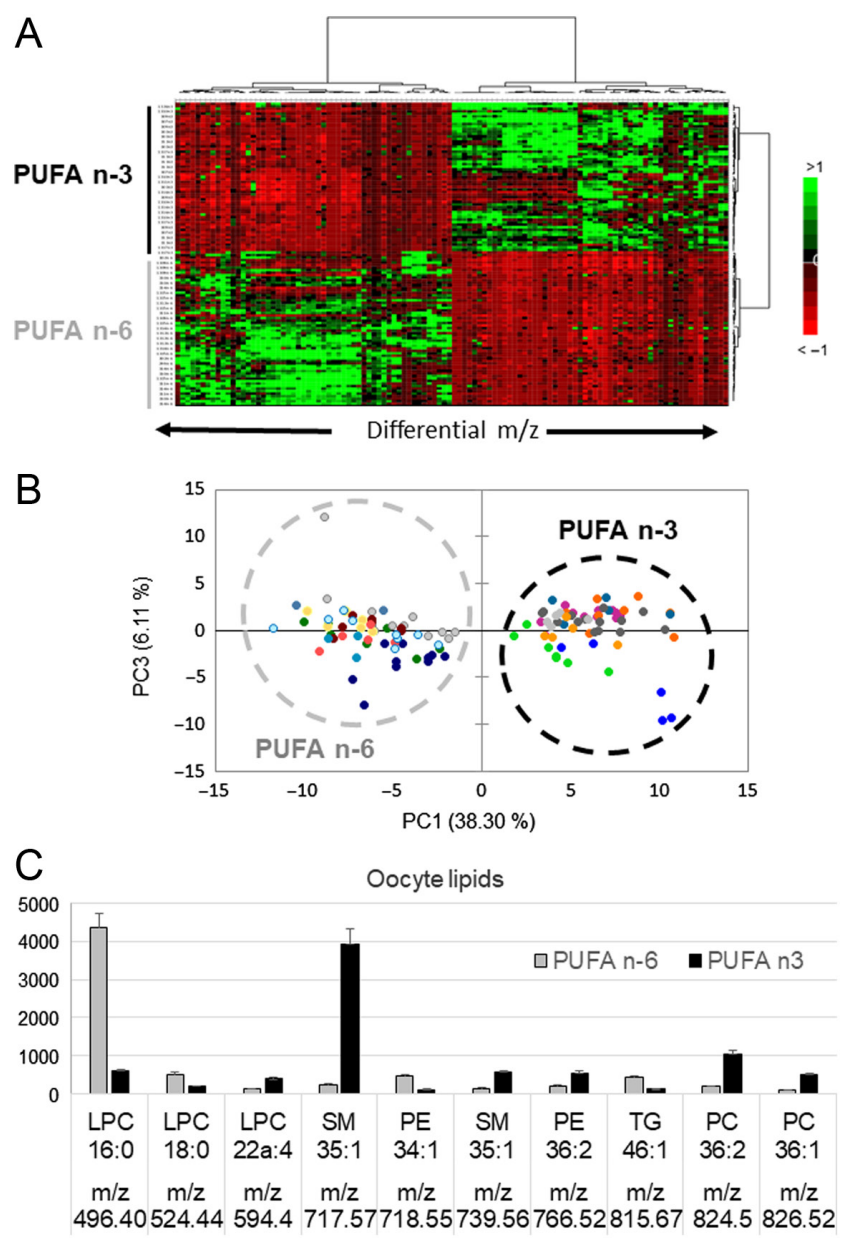

Figure 4 Oocyte lipid composition. Sixty oocytes and 61 oocytes, originating from 9 n-3 cows and 9 n- 6 cows, respectively, underwent individual lipid analysis by MALDI-TOF mass spectrometry.

(A) Hierarchical clustering representing the $110 \mathrm{~m} / \mathrm{z}$ that exhibited a differential abundance between n-3 and n- 6 oocytes (higher abundance represented in green and lower abundance in red). (B) Principle component analysis performed on n-3 and n-6 oocytes. (C) Examples of identified lipids that were either upregulated or downregulated in n-3 oocytes. LPC, lyso-phosphatidylcholine; $\mathrm{SM}$, sphingomyelins; PC, phosphatidylcholines; PE,

phosphatidylethanolamines; TG, triacylglycerols.

composition (Fig. 4B). Among these 110 differential $\mathrm{m} / \mathrm{z}, 42$ lipid species were identified (Supplementary Table 4). Among them, 12 phosphatidylcholines (PC), three phosphatidylethanolamines (PE, C36), two sphingomyelins (SM, C35) and lyso-phosphatidylcholine LPC 22:4 were more abundant in n-3 oocytes, whereas 15 PC, PE 30:0, SM 34:1, two LPC (16:0 and 18:0) and two triacylglycerols $(46: 1,47: 1)$ were more abundant in n-6 oocytes.

\section{Discussion}

This present work is the first, to our knowledge, to assess whether the effects of a n-3 diet on in vitro embryo production in dairy cows still occurred after ovarian stimulation, compared to control cows supplemented with a n-6 diet; it was also the first to assess the effects of several supplementation durations. The results suggest that $\mathrm{n}-3$ supplementation could enhance embryo quality, even with a relatively short supplementation duration of 2-5 weeks.

Supplementation of the diet with n-3 PUFA led to an increase in the Q1-Q2 blastocyst rate, even when compared to control cows supplemented with n-6 PUFA and even after ovarian stimulation. Such supplementation could therefore be a benefit to the in vitro embryo production field. This increase in goodquality embryos is consistent with previous studies reporting a positive effect of $\mathrm{n}-3$ supplementation on conception or gestation rates (for a review Moallem 2018). Indeed, n-3 supplementation seemed to enhance gestation rate (Ambrose et al. 2006, Petit \& Twagiramungu 2006, Dirandeh et al. 2013, Sinedino et al. 2017), partly through enhancing embryo implantation (Mattos et al. 2003), but also by affecting oocyte quality (Moallem et al. 2013, Elis et al. 2016b). Moreover, other studies were performed on embryo production, without ovarian stimulation treatment. An increase in the cleavage rate was reported after flaxseed (Zachut et al. 2010) and fish oil supplementation (Moallem et al. 2013). These results were also consistent with in vitro studies in which a supplementation of the IVM medium with n-3 PUFA, therefore over only $24 \mathrm{~h}$, also led to an enhancement of the metaphase 2 oocyte rate and the subsequent cleavage and blastocyst rates (Marei et al. 2009, Oseikria et al. 2016, Elis et al. 2017). In the future, it would be interesting to assess whether coupling the supplementation of the diet of oocyte donor cows and of IVM medium with n-3 PUFA could further improve in vitro embryo production.

The increases in blastocyst rates reported in the present study were only moderate, but these results are nonetheless interesting and consistent with another study also performed after ovarian stimulation (Thangavelu et al. 2007). In such conditions, one could expect a loss of this effect, due to the strong ovarian stimulation effect of hormonal treatment. The observation of such an effect of $\mathrm{n}-3$ supplementation is thus an interesting result that is encouraging for its use in embryo production or for the improvement of the fertility of dairy cows. Moreover, the control used in the present study was n-6 supplementation, which is the optimal control to evaluate a specific effect of n-3 PUFA. Indeed, when comparing the effect of n-3 PUFA to a saturated FA control, any reported difference could be due to the fact that a saturated FA is being compared to a PUFA, but it would not be possible to prove that it is due to the specific effect of $n-3$ PUFA. On the other hand, the n-6 PUFA control exhibited similar effects as n-3 PUFA (Leroy et al. 2014); it is therefore more difficult to prove a significant difference between the $n-6$ and $n-3$ groups of cows. Previous studies (cited above) reported that 
cleavage rates increase in n-3 PUFA groups compared to saturated controls (Zachut et al. 2010, Moallem et al. 2013), but they also reported an absence of a difference when compared to a n-6 PUFA group (Zachut et al. 2010). Moreover, another study reported a difference in the number of blastomeres of transferable embryo after PUFA supplementation compared to saturated supplementation, but it was not possible to highlight such difference between n-3 and n- 6 supplementation groups (Thangavelu et al. 2007).

In the present study, no difference in the number of puncturedfollicleswasshown. Thiswasexpectedbecause, even if n-3 PUFA supplementation has been reported to increase follicular population (Petit \& Twagiramungu 2002, Bilby et al. 2006, Zachut et al. 2010), this same effect is also observed with n-6 PUFA supplementation (for a review, Leroy et al. 2014). Moreover, none of the embryo production parameters measured at the fourth OPU (after 7 weeks of supplementation) were different between n-3 PUFA and n-6 PUFA cows. Indeed, after 7 weeks of supplementation, a significant increase in plasma n-6 PUFA was reported in the FA composition of the control n- 6 compared to the n-3 PUFA cows. This increase might suggest that the beneficial effects of $n-6$ PUFA on embryo production could explain why control n-6 PUFA embryo rates matched the embryo rates of the n-3 PUFA group, rendering it impossible to find a difference between the groups. Even though the addition of a third experimental group of cows (consisting of a saturated FA diet as a control) was not possible in our conditions, a comparison with this third group would have highlighted the obtained results.

Concerning the duration of n-3 PUFA supplementation required to observe a beneficial effect, the present study suggests even if it has to be further confirmed, that a short duration of supplementation (around 2-5 weeks) could be long enough to observe an improvement in embryo production. Indeed, in our study, a longer period of supplementation did not lead to higher embryo rates. A short period of supplementation means that such supplementation could be applied to the field of embryo production, by limiting the cost of supplementation (3.6€/day/cow in average in our study). Moreover, in vitro results suggested that improvement of blastocyst rates was especially observed in low-quality oocytes (Oseikria et al. 2016, Elis et al. 2017); it is therefore possible that such improvements would not be observed in high-quality oocytes. In addition, the positive effects on embryo rates have not always been reported, i.e. when working with oocyte donor heifers rather than cows in lactation (Ponter et al. 2012). Discrepancies could be attributed to differences in the amount or form of PUFA supplementation, but might also be attributed to the quality of the recovered oocytes. Therefore, the type of supplementation would be especially recommended when working with high genetic merit cows with unpredictable oocyte quality.
The increases in EPA, DHA and total n-3 PUFA percentages in plasma and/or follicular fluid fatty acid composition allowed for the validation of the efficiency of such supplementation in dairy cows, which is in line with our previous study (Elis et al. 2016b). The present study also showed that fatty acid composition variations observed in the plasma were similar to variations observed in the follicular fluid, which is consistent with the literature (Childs et al. 2008, Moallem et al. 2013). These variations needed a longer period of supplementation to appear in follicular fluid compared to plasma, where they were demonstrated after 2 weeks of supplementation.

To ensure that our results were not biased, we also studied physiological measurements. Indeed, differences in MY or EB could contribute to differences in embryo quality. In our study, n-3 PUFA supplementation was not associated with differences in BW, MY, DMI or EB nor with metabolic differences in plasma NEFA, glucose or urea between the two groups of cows; this is consistent with our previous data reporting the effect of this type of diet in dairy cows (Elis et al. 2016b). Indeed, the supplementation was isoenergetic between n-3 and control n- 6 groups, and moderate $(1 \% \mathrm{DM})$, and we therefore did not report the physiological variations that could be observed with supplementations at higher doses or in a different form, such as a reduction in DMI and an increase in MY (Leroy et al. 2014). The absence of metabolic differences, which are in line with previous studies for NEFA (Mattos et al. 2004, Mashek et al. 2005) and plasma glucose (Ambrose et al. 2006) levels, are also probably due to the moderate quantity ( $1 \% \mathrm{DM})$ distributed.

Finally, an increase in the recovered COC rate was reported in n-3 PUFA cows. Even if we have no direct explanation for this finding, the high number of follicles punctured suggests that this difference is real and asks the hypothesis of physical and structural changes between the COC of both groups. Indeed, the lipidomic analyses of the oocytes showed changes mostly in phosphoglycerolipid compositions (mostly PC, 12/18 upregulated lipids and 15/24 downregulated lipids in $\mathrm{n}-3$ oocytes) and SM, with some lipids being upregulated and others being downregulated in n-3 oocytes. PC lipids are a major component of the cell membrane, and SMs are involved in membrane structure stabilisation (Zheng et al. 2006); therefore, these changes could potentially be linked to cell membrane composition and could be also linked to increased elasticity or suction resistance, allowing for an increased COC recovered rate (observed in $\mathrm{n}-3$ group). Such changes in membrane composition were previously reported (Calder 2015). Moreover, less TG (mainly involved in energy storage) was reported in n-3 oocytes, which is consistent with previously reported results suggesting that $\mathrm{n}-3$ supplementation leads to increased lipolysis (Elis et al. 2016a). Further studies are necessary to investigate the relationship between the specific lipids that present a change in their composition and an enhanced oocyte quality. 


\section{Supplementary data}

This is linked to the online version of the paper at https://doi.org/10.1530/REP-18-0644.

\section{Declaration of interest}

The authors declare that there is no conflict of interest that could be perceived as prejudicing the impartiality of the research reported.

\section{Funding}

This work was financially supported by INRA and 'CentreVal de Loire' Region (Bovoméga3 project, APR 2014). This work was financed by European Regional Development Fund (ERDF), the Val-de-Loire Region general comity, the French National Institute for Agricultural Research (INRA) and the French National Institute of Health and Medical Research (INSERM) in the frame of the project SMHART (N³069, LTQ Velos Orbitrap high-resolution mass spectrometer acquisition).

\section{Acknowledgements}

The authors would like to thank the technical staff of the experimental herd INRA UEPAO in Nouzilly (Mickael Delanoue, Christophe Mouaze, Ludovic Métivier and Damien Capo). The authors also acknowledge Bénédicte Grimard at the National Veterinary School of Alfort (ENVA) for helpful scientific discussions and Sophie Daré (from INRA UMR PEGASE, Saint-Gilles) for the NEFA, glucose and urea assays.

\section{References}

Abughazaleh AA, Potu RB \& Ibrahim S 2009 Short communication: the effect of substituting fish oil in dairy cow diets with docosahexaenoic acid-micro algae on milk composition and fatty acids profile. Journal of Dairy Science 92 6156-6159. (https://doi.org/10.3168/jds.2009-2400)

Ambrose DJ, Kastelic JP, Corbett R, Pitney PA, Petit HV, Small JA \& Zalkovic P 2006 Lower pregnancy losses in lactating dairy cows fed a diet enriched in alpha-linolenic acid. Journal of Dairy Science $\mathbf{8 9}$ 3066-3074. (https://doi.org/10.3168/jds.S0022-0302(06)72581-4)

Bertevello PS, Teixeira-Gomes AP, Seyer A, Vitorino Carvalho A, Labas V, Blache MC, Banliat C, Cordeiro LAV, Duranthon V, Papillier P et al. 2018 Lipid identification and transcriptional analysis of controlling enzymes in bovine ovarian follicle. International Journal of Molecular Sciences 19 3261. (https://doi.org/10.3390/ijms19103261)

Bilby TR, Sozzi A, Lopez MM, Silvestre FT, Ealy AD, Staples CR \& Thatcher WW 2006 Pregnancy, bovine somatotropin, and dietary n-3 fatty acids in lactating dairy cows: I. Ovarian, conceptus, and growth hormone-insulin-like growth factor system responses. Journal of Dairy Science 89 3360-3374. (https://doi.org/10.3168/jds.S00220302(06)72373-6)

Butler WR 2000 Nutritional interactions with reproductive performance in dairy cattle. Animal Reproduction Science 60-61 449-457. (https://doi. org/10.1016/S0378-4320(00)00076-2)

Calder PC 2012 Mechanisms of action of (n-3) fatty acids. Journal of Nutrition 142 592S-599S. (https://doi.org/10.3945/jn.111.155259)

Calder PC 2015 Marine omega-3 fatty acids and inflammatory processes: effects, mechanisms and clinical relevance. Biochimica et Biophysica Acta 1851 469-484. (https://doi.org/10.1016/j.bbalip.2014.08.010)

Childs S, Hennessy AA, Sreenan JM, Wathes DC, Cheng Z, Stanton C, Diskin MG \& Kenny DA 2008 Effect of level of dietary n-3 polyunsaturated fatty acid supplementation on systemic and tissue fatty acid concentrations and on selected reproductive variables in cattle. Theriogenology 70 595-611. (https://doi.org/10.1016/j. theriogenology.2008.04.002)

Dirandeh E, Towhidi A, Zeinoaldini S, Ganjkhanlou M, Ansari Pirsaraei Z \& Fouladi-Nashta A 2013 Effects of different polyunsaturated fatty acid supplementations during the postpartum periods of early lactating dairy cows on milk yield, metabolic responses, and reproductive performances. Journal of Animal Science 91 713-721. (https://doi. org/10.2527/jas.2012-5359)

Elis S, Desmarchais A, Freret S, Maillard V, Labas V, Cognié J, Briant E, Hivelin C, Dupont J \& Uzbekova S 2016a Effect of a long-chain n-3 polyunsaturated fatty acid-enriched diet on adipose tissue lipid profiles and gene expression in Holstein dairy cows. Journal of Dairy Science 99 10109-10127. (https://doi.org/10.3168/jds.2016-11052)

Elis S, Freret S, Desmarchais A, Maillard V, Cognié J, Briant E, Touzé JL, Dupont M, Faverdin P, Chajès V et al. 2016b Effect of a long chain n-3 PUFA-enriched diet on production and reproduction variables in Holstein dairy cows. Animal Reproduction Science 164 121-132. (https://doi.org/10.1016/j.anireprosci.2015.11.020)

Elis S, Oseikria M, Vitorino Carvalho A, Bertevello PS, Corbin E, TeixeiraGomes AP, Lecardonnel J, Archilla C, Duranthon V, Labas V et al. 2017 Docosahexaenoic acid mechanisms of action on the bovine oocytecumulus complex. Journal of Ovarian Research 10 74. (https://doi. org/10.1186/s13048-017-0370-z)

INRA 2018 Alimentation des Ruminants: Apports Nutritionnels - Besoins et Réponses des Animaux - Rationnement - Tables des Valeurs des Aliments. Paris, France: Quae Editions.

International Embryo Transfer Society 2013 In Manual of the International Embryo Transfer Society: A Procedural Guide and General Information for the Use of Embryo Transfer Techology Emphasizing Sanitary Procedures, 4th ed. Eds DA Stringfellow \& MD Givens. International Embryo Transfer Society: Champaign, Illinois, USA.

Lefils J, Géloën A, Vidal H, Lagarde M \& Bernoud-Hubac N 2010 Dietary DHA: time course of tissue uptake and effects on cytokine secretion in mice. British Journal of Nutrition 104 1304-1312. (https://doi. org/10.1017/S0007114510002102)

Leroy JL, Vanholder T, Van Knegsel AT, Garcia-Ispierto I \& Bols PE 2008 Nutrient prioritization in dairy cows early postpartum: mismatch between metabolism and fertility? Reproduction in Domestic Animals 43 (Supplement 2) 96-103. (https://doi.org/10.1111/j.1439-0531.2008.01148.x)

Leroy JL, Sturmey RG, Van Hoeck V, De Bie J, Mckeegan PJ \& Bols PEJ 2014 Dietary fat supplementation and the consequences for oocyte and embryo quality: hype or significant benefit for dairy cow reproduction? Reproduction in Domestic Animals 49 353-361. (https://doi.org/10.1111/ rda.12308)

Marei WF, Wathes DC \& Fouladi-Nashta AA 2009 The effect of linolenic acid on bovine oocyte maturation and development. Biology of Reproduction 81 1064-1072. (https://doi.org/10.1095/biolreprod.109.076851)

Mashek DG, Bertics SJ \& Grummer RR 2005 Effects of intravenous triacylglycerol emulsions on hepatic metabolism and blood metabolites in fasted dairy cows. Journal of Dairy Science 88 100-109. (https://doi. org/10.3168/jds.S0022-0302(05)72667-9)

Mattos R, Guzeloglu A, Badinga L, Staples CR \& Thatcher WW 2003 Polyunsaturated fatty acids and bovine interferon-tau modify phorbol ester-induced secretion of prostaglandin F2 alpha and expression of prostaglandin endoperoxide synthase-2 and phospholipase-A2 in bovine endometrial cells. Biology of Reproduction 69 780-787. (https:// doi.org/10.1095/biolreprod.102.015057)

Mattos R, Staples CR, Arteche A, Wiltbank MC, Diaz FJ, Jenkins TC \& Thatcher WW 2004 The effects of feeding fish oil on uterine secretion of PGF2alpha, milk composition, and metabolic status of periparturient Holstein cows. Journal of Dairy Science 87 921-932. (https://doi. org/10.3168/jds.S0022-0302(04)73236-1)

Moallem U 2018 Invited review: roles of dietary n-3 fatty acids in performance, milk fat composition, and reproductive and immune systems in dairy cattle. Journal of Dairy Science 101 8641-8661. (https:// doi.org/10.3168/jds.2018-14772)

Moallem U, Shafran A, Zachut M, Dekel I, Portnick Y \& Arieli A 2013 Dietary alpha-linolenic acid from flaxseed oil improved folliculogenesis and IVF performance in dairy cows, similar to eicosapentaenoic and docosahexaenoic acids from fish oil. Reproduction 146 603-614. (https://doi.org/10.1530/REP-13-0244) 
Oseikria M, Elis S, Maillard V, Corbin E \& Uzbekova S 2016 N-3 polyunsaturated fatty acid DHA during IVM affected oocyte developmental competence in cattle. Theriogenology 85 1625.e2-1634. e2. (https://doi.org/10.1016/j.theriogenology.2016.01.019)

Petit HV \& Twagiramungu H 2006 Conception rate and reproductive function of dairy cows fed different fat sources. Theriogenology $\mathbf{6 6}$ 1316-1324. (https://doi.org/10.1016/j.theriogenology.2006.04.029)

Petit HV, Dewhurst RJ, Scollan ND, Proulx JG, Khalid M, Haresign W, Twagiramungu H \& Mann GE 2002 Milk production and composition, ovarian function, and prostaglandin secretion of dairy cows fed omega-3 fats. Journal of Dairy Science 85 889-899. (https://doi.org/10.3168/jds. S0022-0302(02)74147-7)

Plourde M \& Cunnane SC 2007 Extremely limited synthesis of long chain polyunsaturates in adults: implications for their dietary essentiality and use as supplements. Applied Physiology, Nutrition, and Metabolism 32 619-634. (https://doi.org/10.1139/H07-034)

Ponter AA, Guyader-Joly C, Nuttinck F, Grimard B \& Humblot P 2012 Oocyte and embryo production and quality after OPU-IVF in dairy heifers given diets varying in their $n-6 / n-3$ fatty acid ratio. Theriogenology 78 632-645. (https://doi.org/10.1016/j.theriogenology.2012.03.009)

Rico C, Fabre S, Medigue C, Di Clemente N, Clement F, Bontoux $M$, Touze JL, Dupont M, Briant E, Remy B et al. 2009 Anti-Mullerian hormone is an endocrine marker of ovarian gonadotropin-responsive follicles and can help to predict superovulatory responses in the cow. Biology of Reproduction 80 50-59. (https://doi.org/10.1095/biolreprod.108.072157)

Roche JF 2006 The effect of nutritional management of the dairy cow on reproductive efficiency. Animal Reproduction Science 96 282-296. (https://doi.org/10.1016/j.anireprosci.2006.08.007)

SAS Institute Inc. 2013 SAS/Stat 9.2 User's Guide, 2nd ed. Cary, NC: SAS Institute Incorporated.

Simopoulos AP 2002 Omega-3 fatty acids in inflammation and autoimmune diseases. Journal of the American College of Nutrition 21 495-505. (https://doi.org/10.1080/07315724.2002.10719248)

Sinedino LDP, Honda PM, Souza LRL, Lock AL, Boland MP, Staples CR, Thatcher WW \& Santos JEP 2017 Effects of supplementation with docosahexaenoic acid on reproduction of dairy cows. Reproduction 153 707-723. (https://doi.org/10.1530/REP-16-0642)

Tessaro FH, Ayala TS \& Martins JO 2015 Lipid mediators are critical in resolving inflammation: a review of the emerging roles of eicosanoids in diabetes mellitus. BioMed Research International 2015 568408. (https:// doi.org/10.1155/2015/568408)

Thangavelu G, Colazo MG, Ambrose DJ, Oba M, Okine EK \& Dyck MK 2007 Diets enriched in unsaturated fatty acids enhance early embryonic development in lactating Holstein cows. Theriogenology 68 949-957. (https://doi.org/10.1016/j.theriogenology.2007.07.002)

Urlep Z \& Rozman D 2013 The interplay between circadian system, cholesterol synthesis, and steroidogenesis affects various aspects of female reproduction. Frontiers in Endocrinology 4 111. (https://doi. org/10.3389/fendo.2013.00111)

Zachut M, Dekel I, Lehrer H, Arieli A, Arav A, Livshitz L, Yakoby S \& Moallem U 2010 Effects of dietary fats differing in n-6:n-3 ratio fed to high-yielding dairy cows on fatty acid composition of ovarian compartments, follicular status, and oocyte quality. Journal of Dairy Science 93 529-545. (https://doi.org/10.3168/jds.2009-2167)

Zheng W, Kollmeyer J, Symolon H, Momin A, Munter E, Wang E, Kelly S, Allegood JC, Liu Y, Peng Q et al. 2006 Ceramides and other bioactive sphingolipid backbones in health and disease: lipidomic analysis, metabolism and roles in membrane structure, dynamics, signaling and autophagy. Biochimica and Biophysica Acta 1758 1864-1884. (https:// doi.org/10.1016/j.bbamem.2006.08.009)

Received 21 December 2018

First decision 26 February 2019

Revised manuscript received 16 April 2019

Accepted 23 April 2019 